\title{
AUTHOR BLOCK
}

Audrey Y Chu ${ }^{1,2,48}$, Xuan Deng ${ }^{3,48}$, Virginia A Fisher ${ }^{3,48}$, Alexander Drong ${ }^{4}$, Yang Zhang ${ }^{5,6}$, Mary 6 F Feitosa $^{7}$, Ching-Ti Liu ${ }^{3}$, Olivia Weeks ${ }^{6}$, Audrey C Choh ${ }^{8}$, Qing Duan ${ }^{9}$, Thomas D Dyer ${ }^{10}$, John 7 D Eicher ${ }^{1}$, Xiuqing Guo ${ }^{11}$, Nancy L Heard-Costa ${ }^{3}$, Tim Kacprowski ${ }^{12,13}$, Jack W Kent Jr ${ }^{14}$, Leslie 8 A Lange ${ }^{9}$, Xinggang Liu ${ }^{15}$, Kurt Lohman ${ }^{16,17}$, Lingyi Lu ${ }^{17}$, Anubha Mahajan ${ }^{4}$, Jeffrey R 9 O'Connell $^{15}$, Ankita Parihar ${ }^{15}$, Juan M Peralta ${ }^{10}$, Albert V Smith ${ }^{18,19}$, Yi Zhang ${ }^{20}$, Georg Homuth ${ }^{12}$, Ahmed H Kissebah ${ }^{20,49}$, Joel Kullberg ${ }^{21}$, René Laqua ${ }^{22}$, Lenore $\mathrm{J} \mathrm{Launer}^{23}$, Matthias Nauck ${ }^{24,13}$, Michael Olivier ${ }^{14,20}$, Patricia A Peyser ${ }^{25}$, James G Terry ${ }^{26}$, Mary K Wojczynski ${ }^{7}$, Jie Yao ${ }^{11}$, Lawrence F Bielak $^{25}$, John Blangero ${ }^{10}$, Ingrid B Borecki ${ }^{7}$, Donald W Bowden ${ }^{27,28}$, John Jeffrey Carr $^{26}$, Stefan A Czerwinski ${ }^{29}$, Jingzhong Ding ${ }^{16,30}$, Nele Friedrich ${ }^{24,13}$, Vilmunder Gudnason ${ }^{18,19}$, Tamara B Harris $^{23}$, Erik Ingelsson ${ }^{31,32}$, Andrew D Johnson ${ }^{1}$, Sharon LR Kardia ${ }^{25}$, Carl D

15 Langefeld ${ }^{17}$, Lars Lind ${ }^{21}$, Yongmei Liu ${ }^{16,33}$, Braxton D Mitchell ${ }^{15,34}$, Andrew P Morris ${ }^{35,4}$, Thomas H Mosley Jr ${ }^{36}$, Jerome I Rotter ${ }^{11}$, Alan R Shuldiner ${ }^{15}$, Bradford Towne ${ }^{8}$, Henry Völzke ${ }^{37,13,38}$, Henri Wallaschofski ${ }^{24}$, James $G$ Wilson ${ }^{39}$, Matthew Allison ${ }^{40}$, Cecilia M Lindgren ${ }^{41}$, Wolfram Goessling $^{6,42,43,44,45}$, L Adrienne Cupples ${ }^{1,3,50}$, Matthew L Steinhauser ${ }^{5,6,45,46,50}$, Caroline S Fox $1,47,50$

\section{AFFILIATIONS}

1. NHLBI's Framingham Heart Study, Framingham MA USA

2. Division of Preventive Medicine, Brigham and Women's Hospital and Harvard Medical School, Boston MA USA

3. Department of Biostatistics, Boston University School of Public Health, Boston MA USA

4. Wellcome Trust Centre for Human Genetics, University of Oxford, Oxford UK

5. Department of Medicine, Brigham and Women's Hospital and Harvard Medical School, Boston MA USA

6. Division of Genetics, Brigham and Women's Hospital and Harvard Medical School, Boston MA USA

7. Department of Genetics, Washington University, St. Louis MO USA

8. Division of Epidemiology and Biostatistics, Department of Population and Public Health Sciences, Wright State University Boonshoft School of Medicine, Dayton OH USA

9. Department of Genetics, University of North Carolina, Chapel Hill NC USA

10. South Texas Diabetes and Obesity Institute, University of Texas Health Science Center at San Antonio \& University of Texas of the Rio Grande Valley, Brownsville TX USA

11. Institute for Translational Genomics and Population Sciences, Department of Pediatrics, LABioMed at Harbor-UCLA Medical Center, Torrance CA USA 
41 12. Interfaculty Institute for Genetics and Functional Genomics, University Medicine

42 Greifswald, Greifswald Germany

43 13. German Centre for Cardiovascular Research (DZHK), Partner Site Greifswald, Germany

44 14. TOPS Nutrition and Obesity Research Center, Department of Genetics, Texas

Biomedical Research Institute, San Antonio TX USA

15. University of Maryland School of Medicine, Baltimore MD USA

16. Wake Forest School of Medicine, Winston-Salem NC USA

17. Department of Biostatistical Sciences, Wake Forest School of Medicine, Winston-Salem NC USA

18. Icelandic Heart Association, Kopavogur Iceland

19. Faculty of Medicine, University of Iceland, Reykjavik Iceland

20. TOPS Obesity and Metabolic Research Center, Biotechnology and Bioengineering Center, Department of Physiology at the Medical College of Wisconsin, WI USA

21. Department of Surgical Sciences, Section of Radiology, Uppsala University, Uppsala Sweden

22. Department of Neuroradiology, University Hospital Berne, Berne Switzerland

23. National Institute on Aging, Intramural Research Program, National Institutes of Health, Bethesda MD USA

24. Institute for Clinical Chemistry and Laboratory Medicine, University Medicine Greifswald, Greifswald Germany

25. Department of Epidemiology, School of Public Health, University of Michigan, Ann Arbor MI USA

26. Departments of Radiology and Radiologic Sciences, Cardiovascular Medicine and Biomedical Informatics, Vanderbilt University Medical Center, Nashville TN USA

27. Center for Genomics and Personalized Medicine Research, Wake Forest University Health Sciences, Winston-Salem NC USA

28. Department of Biochemistry, Center for Diabetes Research, and Center for Human Genomics, Wake Forest University School of Medicine, Winston-Salem NC USA

29. Department of Epidemiology, Human Genetics and Environmental Sciences, University of Texas Health Science Center (UTHealth) School of Public Health Brownsville Campus, Brownsville TX USA

30. Gerontology and Geriatric Medicine, Wake Forest School of Medicine, Winston-Salem NC USA

31. Department of Medical Sciences, Molecular Epidemiology and Science for Life Laboratory, Uppsala University, Uppsala Sweden

32. Department of Medicine, Division of Cardiovascular Medicine, Stanford University School of Medicine, Stanford CA USA

33. Department of Epidemiology and Prevention, Wake Forest School of Medicine, WinstonSalem NC USA

34. Geriatrics Research and Education Clinical Center, Baltimore Veterans Administration Medical Center, Baltimore MD USA

35. Department of Biostatistics, University of Liverpool, Liverpool UK

36. University of Mississippi Medical Center, Jackson MS USA

37. Institute for Community Medicine, University Medicine Greifswald, Greifswald Germany 
38. German Centre for Diabetes Research (DZD), Site Greifswald, Germany

39. Department of Physiology and Biophysics, University of Mississippi Medical Center,

40. Division of Preventive Medicine, Department of Family Medicine and Public Health, UC

41. Li Ka Shing Centre for Health Information and Discovery, The Big Data Institute,

42. Harvard Stem Cell Institute, Cambridge MA USA

43. Gastroenterology Division, Brigham and Women's Hospital, Harvard Medical School,

94

44. Dana-Farber Cancer Institute, Boston MA USA

45. Broad Institute of MIT and Harvard, Cambridge MA USA

46. Division of Cardiovascular Medicine, Brigham and Women's Hospital and Harvard Medical School, Boston MA USA

47. Division of Endocrinology, Brigham and Women's Hospital and Harvard Medical School, Boston MA USA

48. These authors contributed equally to this work

49. This author is deceased

50. These authors jointly supervised this work

Correspondence should be addressed to AYC (audrey.chu@nih.gov), MLS

(msteinhauser@partners.org),or CSF (foxca@nhlbi.nih.gov)

\section{ADDRESSES FOR CORRESPONDENCE:}

Audrey Y Chu, PHD

NHLBl's Framingham Heart Study

Framingham MA 01702 USA

audrey.chu@nih.gov

Matthew L. Steinhauser, MD

Brigham and Women's Hospital and Harvard Medical School

Boston MA 02115 USA

msteinhauser@partners.org

Caroline S Fox, MD MPH

NHLBl's Framingham Heart Study

Framingham MA 01702 USA

foxca@nhlbi.nih.gov

KEY WORDS: GWAS, obesity, ectopic fat, adipocyte development, differentiation

WORD COUNT: intro paragraph (156); main text (2335) 


\section{INTRODUCTORY PARAGRAPH}

130 Variation in body fat distribution contributes to the metabolic sequelae of obesity. The genetic

131 determinants of body fat distribution are poorly understood. The goal of this study was to gain

132 new insights into the underlying genetics of body fat distribution by conducting sample-size

133 weighted fixed-effects genome-wide association meta-analyses in up to 9,594 women and

1348,738 men for six ectopic fat traits in European, African, Hispanic, and Chinese ancestry

135 populations, with and without sex stratification. In total, 7 new loci were identified in association

136 with ectopic fat traits (ATXN1, UBE2E2, EBF1, RREB1, GSDMB, GRAMD3 and ENSA; $\mathrm{P}<5 \times 10^{-}$

137 8; FDR $<1 \%$ ). Functional analysis of these genes revealed that loss of function of both ATXN1

138 and UBE2E2 in primary mouse adipose progenitor cells impaired adipocyte differentiation,

139 suggesting a physiological role for $A T X N 1$ and UBE2E2 in adipogenesis. Future studies are

140 necessary to further explore the mechanisms by which these genes impact adipocyte biology

141 and how their perturbations contribute to systemic metabolic disease. 
Variation in body fat distribution is associated with cardiometabolic risk, including

144 diabetes, hypertension and coronary heart disease,$^{1-5}$ and is at least partially independent of 145 total adiposity. Adipose tissue can be quantified non-invasively using computed tomography

$146(\mathrm{CT})$ and magnetic-resonance imaging (MRI) to measure fat volume and fat attenuation in

147 different tissue compartments. We previously demonstrated that both indices, in addition to

148 relative fat distribution, are important predictors of cardiometabolic risk. ${ }^{6-11}$

149 Several lines of evidence suggest a unique genetic component to body fat distribution.

150 First, indices of body fat distribution are heritable with values ranging from $36-47 \%$, even after

151 adjustment for body mass index (BMI). ${ }^{12}$ Second, unique genetic loci exist for body fat

152 distribution. For example, we identified a SNP associated with pericardial fat ${ }^{13}$ that was not

153 associated with visceral fat, ${ }^{12}$ BMI or waist-hip-ratio (WHR). ${ }^{14,15}$ Third, several lipodystrophy

154 syndromes, characterized by abnormal body fat distribution, are genetically mediated. ${ }^{16}$

155 The current study presents a genome-wide association study and meta-analysis of

156 adipose tissue traits derived from imaging biomarkers (Supplementary Table 1) from 2.6 million

157 SNPs in up to 9,594 women and 8,738 men of European, African, Hispanic and Chinese

158 ancestry (see Supplementary Tables 2, 3 and 4) and uses mouse models to characterize 159 selected loci.

160 Subcutaneous and visceral adipose tissue (SAT, VAT) were previously estimated to 161 have heritabilities of $57 \%$ and $36 \%$, respectively ${ }^{12,17}$ (Supplementary Table 5 ). To assess the 162 genetic contribution to variation in fat attenuation traits, which serve as indirect markers of fat 163 quality (SAT Hounsfield Units [SATHU] and VATHU), heritability $\left(\mathrm{H}^{2}\right)$ was estimated in 3,312 164 women and men in the Framingham Heart Study (FHS), and found to be between 29-31\% $165\left(\mathrm{P}<1 \times 10^{-15}\right)$. To assess the shared genetic contribution between ectopic fat traits, the genetic 166 correlations were estimated among 3,336 women and men in FHS. Moderate to strong 167 statistically significant correlations were observed between almost all ectopic fat traits pairs 
168 ( 0.35 to 0.67 and -0.74 to -0.35 , all $P<5 \times 10^{-4}$; Supplementary Table 6 ), suggesting shared loci 169 between ectopic fat traits. However, not all genes were shared between traits $\left(P<5 \times 10^{-11}\right.$ for 170 non-overlapping correlations for all pairwise comparisons). The genetic correlations across the 171 ectopic fat traits are also reflected in the phenotypic correlations (Supplementary Table 7). 172 In this combined multiethnic sample-size weighted fixed-effects meta-analysis ${ }^{18,19}$ of up 173 to 18,332 participants, a total of 11 locus-trait associations ( 7 novel and 4 known) attained 174 genome-wide significance $\left(P<5 \times 10^{-8}\right)$ out of 27 genomic scans (from analysis of 9 traits and 175 models in 3 strata - overall, women and men). Of the 7 novel loci, 3 were associated with 176 volumetric subcutaneous (GSDMB) and visceral fat traits (GRAMD3 and RREB1), 2 were 177 associated with pericardial fat (ENSA and EBF1), 1 was associated with fat attenuation 178 (ATXN1), and 1 was associated with relative fat distribution (VAT/SAT ratio [UBE2E2]) (Table 1; 179 Supplementary Figures 1a-g; with imputation quality in Supplementary Table 8). Associations 180 were robust across ancestry-stratified sensitivity analyses (Supplementary Figures 2a-g and 3a181 g; Supplementary Table 9). Manhattan plots and QQ plots for each analysis showed minimal 182 inflation of association test statistics (Supplementary Figures 4a-g). The remaining 4 loci 183 (LYPLAL1, LY86, FTO, TRIB2) attaining genome-wide significance were previously 184 identified. ${ }^{12,13}$ rs2123685, located between the 3' untranslated regions of ZPBP2 and GSDMB, was associated with SAT in women only $\left(P_{\text {women }}=3.4 \times 10^{-8}\right.$, Supplementary Table 10a). Investigation 187 of related ectopic traits among women revealed a direction-consistent nominal association with 188 VAT $\left(\mathrm{P}=4.8 \times 10^{-4}\right)$. SNPs at $F T O$, the canonical-BMI locus, attained genome-wide significance 189 in association with SAT in the overall sample $\left(P=1.4 \times 10^{-9}\right)$.

190 The newly identified association at $R R E B 1$ with VATadjBMI ( $\mathrm{rs} 2842895, \mathrm{P}=1.1 \times 10^{-8}$ ) 191 was observed in the overall sample and both sexes (Supplementary Table 10b). Examination 192 of related ectopic traits demonstrated nominal associations with VAT and VAT/SAT ratio adjBMI $193\left(P=4.8 \times 10^{-5}\right.$ and $P=8.9 \times 10^{-6}$ respectively). The newly identified association of $r s 10060123$ near 
194 GRAMD3 for VATadjBMI was specific to women $\left(P=4.5 \times 10^{-8}\right)$. This locus was nominally 195 associated with VAT and VAT/SAT ratio adjBMI in women (Supplementary Table 10c). PAT represents distinct ectopic fat deposition around the heart. Two findings in the overall sample at the ENSA and EBF1 loci $\left(\mathrm{P}=2.8 \times 10^{-9}\right.$ and $1.0 \times 10^{-9}$, respectively, Table 1$)$ have

198 not been previously associated with ectopic fat, general adiposity or body fat distribution.

199 Associations at ENSA and EBF1 did not appear to be sex-specific (Supplementary Tables 10d 200 and 10e). Further investigation of the ENSA and EBF1 loci showed no associations with SAT, 201 VAT or VAT/SAT ratio, underscoring their specificity to PAT. TRIB2 was associated with PAT in 202 this and our prior meta-analysis $\left(\mathrm{P}<5 \times 10^{-8}\right) .^{13}$

203 Cellular characteristics of fat quality, such as lipid content, vascularity, and adipocyte 204 size and number, may be important factors influencing metabolic risk, ${ }^{7,10}$ but direct assessment 205 is invasive. Fat attenuation traits, assessed with computed tomography, are correlated with fat 206 quality characteristics ${ }^{20,21}$ and thus represent indirect markers of fat quality. ATXN1 was 207 associated with SATHU among men only $\left(P=1.4 \times 10^{-8}\right)$ with no association among women $208(P=0.36$, Supplementary Table 10f). Examination of related ectopic fat traits indicated similar 209 direction of association with VATHU, and opposite direction for SAT and VAT (Supplementary 210 Table 10f) which is consistent with epidemiology findings. ${ }^{7}$

211 The ratio of visceral to subcutaneous fat volumes (VAT/SAT ratio) represents the 212 propensity to store fat viscerally. UBE2E2 was associated with VAT/SAT ratio $\left(\mathrm{P}=3.1 \times 10^{-10}\right) ; a$ 213 nominal association was also identified with VAT $\left(P=1.4 \times 10^{-3}\right)$ but not SAT, suggesting the 214 finding is mostly driven by the higher relative abundance of VAT. The direction of association in 215 both sex strata was consistent (Supplementary Table 10g). Two known body fat distribution 216 loci, $L Y P L A L 1$ and $L Y 86$, were also associated with VAT/SAT ratio at genome-wide significance 217 (Table 1), consistent with our prior analyses. ${ }^{12}, 22$ 
Calculation of false discovery rate (FDR) to account for multiple testing across the 27 meta-analyses showed all ectopic fat loci that attained genome-wide significance in each 220 individual GWAS $\left(\mathrm{P}<5 \times 10^{-8}\right)$ also attained an $\mathrm{FDR}<1 \%$.

To examine the association of the 7 newly identified ectopic fat loci with BMI and WHR,

222 cross-trait evaluations for each lead SNP were performed in the most recent GIANT meta-

223 GWAS, with sample sizes 10-20 times larger than the current study. ${ }^{14,15}$ Only 2 out of 14 SNP-

224 trait (BMI or WHR) associations were significant after Bonferroni correction for multiple testing

$225\left(\mathrm{P}<0.05 / 14=3.6 \times 10^{-3}\right.$; Supplementary Table 10a-g), highlighting the specificity and uniqueness

226 of the ectopic fat loci.

227 To evaluate the relationship between the known $97 \mathrm{BMI}$ and $49 \mathrm{WHR}$ loci ${ }^{14,15}$ and

228 ectopic fat traits, we examined the association for these loci with fat volume and relative fat

229 volume traits among the combined multiethnic sample of women and men. Because the

230 ectopic fat data may be underpowered to determine statistically significant results, we

231 hypothesized that the direction of the BMI and WHR findings would be directionally consistent

232 with abdominal ectopic traits, even if the p-values were not significant (Supplementary Table

233 11). Direction consistent SNP-trait associations between SAT and BMI were observed for 87 of

23497 loci $\left(P_{\text {binomial }}=8.9 \times 10^{-17}\right)$. When restricted to the 27 loci nominally associated with SAT

$235\left(\mathrm{P}_{\mathrm{SAT}}<0.05\right)$, all 27 SNP-SAT associations were directionally consistent with BMI

$236\left(\mathrm{P}_{\text {binominal }}=7.5 \times 10^{-9}\right)$. SAT is not an ectopic fat depot and may represent a metabolic sink for

237 healthier fat storage that is highly correlated with BMI and shares genetic risk factors (as shown

238 with the enriched number of direction consistent associations), yet also represents a unique

239 metric of fat distribution with unique genetic influences (as shown with the GSDMB-SAT

240 association). No other traits showed directionally consistent associations with the BMI or WHR

241 (all $\mathrm{P}>0.05$ ). These results further underscore how ectopic fat traits are uniquely disparate traits

242 as compared to BMI and WHR. 
Ectopic fat depots are associated with cardiometabolic risk and cardiovascular events. ${ }^{8-}$

24411 To gain insight into potential mechanisms linking these conditions, we evaluated the

245 association of the new ectopic fat loci with traits from large-scale genetics consortia. Of 66 pairs

246 of lead SNP-trait associations examined, 3 associations (UBE2E2-type 2 diabetes [T2D], EBF1-

247 triglycerides, and EBF1-HDL cholesterol) were statistically significant after Bonferroni correction

248 for multiple testing $\left(P<0.05 / 66=8 \times 10^{-4}\right.$; Supplementary Table 12).

249 To examine if any of the new variants overlap with known regulatory regions in adipose

250 tissue, lead SNPs and variants in linkage disequilibrium (LD) with the lead SNPs $\left(r^{2}>0.8\right)$ were

251 interrogated using ENCODE Consortium data implemented in HaploReg ${ }^{23}$ and RegulomeDB. ${ }^{24}$

252 Except for ATXN1, all other loci contained SNPs in LD with the lead SNP that overlapped with 253 known regulatory regions in adipose tissue. For example, the lead UBE2E2 variant

254 (rs7374732), and other SNPs in LD, overlapped with a known enhancer region in adipose 255 derived stem cells (Supplementary Table 13).

The list of candidate loci was further prioritized based on visual examination of regional

257 association plots (Supplementary Figures $1 \mathrm{a}-\mathrm{g}$ ) and identification of 1) a localized association

258 within a gene body at each locus (RREB1, ATXN1 and UBE2E2), or 2) a localized association

259 near the gene body concomitant with the lack of other genes within $1 \mathrm{Mbp}$ of the lead SNP

260 (EBF1). In applying these criteria, four genes were selected for additional functional study.

261 To test the hypothesis that inter-depot differences in gene expression or their dynamic

262 regulation during adipocyte development would identify candidates with a higher likelihood of

263 functional significance, expression of 4 genes (Ebf1, Rreb1 Atxn1, Ube2e2) in murine SAT,

264 VAT, and PAT depots was assessed by qPCR. Ube2e2 was expressed more highly in the 265 perigonadal VAT of 6 week-old C57BL/6 mice relative to the SAT $(2.1$ fold, $p<0.05, n=5)$ or PAT 266 (2.6 fold, $\mathrm{p}<0.01, \mathrm{n}=5$ ), but no differences were observed for Ebf1, Rreb1 or Atxn1 (Figure 1a).

267 Differential gene expression of these 4 genes was also assessed in murine diet-induced 268 obesity. A 2.1 fold induction of Atxn1 expression in SAT of diet-induced obese mice was 
observed relative to lean controls $(p<0.05, n=6)$. Significant differences were not observed for

270 Ebf1, Rreb1, or Ube2e2 in response to the obesogenic stimulus (Figure 1b).

To explore a potential role for the candidate genes in adipocyte development, we

272 examined their regulation during ex vivo adipogenic differentiation of progenitor-rich stromal-

273 vascular cell fractions isolated from the subcutaneous and visceral depots of C57BL/6 mice.

274 Candidate gene expression was measured at regular intervals during adipogenic differentiation.

275 In progenitors isolated from both VAT and SAT, we observed a significant down-regulation of

276 Atxn1, Ube2e2, and Ebf1 during adipogenesis (Figure 1c and Supplementary Figure 5).

277 However, in all three instances the expression returned to near baseline levels by $96 \mathrm{~h}$ post-

278 adipogenic induction. In contrast, no significant transcriptional regulation of Rreb1 after

279 adipogenic induction was observed (Supplementary Figure 5).

Both Atxn1 and Ube2Ee2 showed evidence of dynamic regulation of gene expression

281 during adipogenesis with variable depot-specific expression in the murine models providing

282 rationale to further explore their functional significance with a genetic loss-of-function assay.

283 Knock-down of both genes with specific shRNA retroviral constructs during ex vivo

284 adipogenesis of SAT progenitors impaired the formation of lipid-containing adipocytes relative to

285 vector control infected cells, whereas only Ube2e2 knock-down impaired adipogenesis in

286 progenitors isolated from VAT (Figure 1d,e).

287 Our findings provide insight into the genetics of body fat distribution. The scant number

288 of significant associations observed between the ectopic fat loci and more general measures of

289 adiposity, such as BMI and WHR, ${ }^{14,15}$ demonstrates the specificity of the ectopic fat

290 associations, highlights the utility of precise phenotyping of fat distribution, and suggests

291 different mechanisms involved in ectopic fat storage compared to more general adiposity

292 measures. This specificity was particularly notable for PAT loci, which demonstrate no

293 associations with SAT, VAT, VAT/SAT ratio, BMI or WHR. 
In addition, few cross-trait associations were observed for ectopic fat loci and other cardiometabolic traits, which is striking given the epidemiologic associations between ectopic fat and cardiometabolic risk ${ }^{1-5}$. One notable exception is UBE2E2, which is a known T2D locus ${ }^{25,26 .}$

297 The lead T2D SNP does not appear to be in LD with the lead SNP from our study $\left(\mathrm{r}^{2}[\mathrm{rs} 7374732\right.$, 298 rs7612463]<0.08 across all HapMap2 populations), and therefore likely represents an

299 independent signal. The major allele at rs7374732 is associated with both lower VAT/SAT ratio 300 and lower risk of T2D, suggesting that targeting relative fat distribution may have beneficial 301 downstream effects.

Functional studies support a physiologic role for UBE2E2 and ATXN1 through regulation 303 of adipocyte differentiation. ATXN1 encodes a chromatin binding factor involved in the 304 repression of Notch signaling. It has been implicated in neurologic diseases, including spinocerebellar ataxia 1, but there are no reported associations between SNPs in ATXN1 and adiposity-related traits. In contrast, UBE2E2 is a known T2D GWAS locus, ${ }^{25-27}$ although the markers are in low LD with the lead SNP in the present study. UBE2E2 (3p24.2) encodes the ubiquitin-conjugating enzyme E2E2, which is expressed in human pancreas, liver, muscle and adipose tissues. The present GWAS results highlight UBE2E2 in association with the VAT/SAT ratio, a measure of the relative propensity to store fat in the visceral cavity rather than the

311 subcutaneous compartment. We therefore speculate that SNP-associated modulation of gene

312 expression or function of the protein products may impact adiposity through an effect on

313 adipocyte differentiation and relative impairments in adipocyte development may partially

314 explain a default propensity to deposit viscerally as compared to subcutaneously. Given the uniqueness of the ectopic fat traits, the sample size was limited in comparison

316 to other meta-analyses. Moreover, identification of candidate genes based on proximity to a 317 GWAS signal may miss long distance interactions between genes and regulatory domains. In 318 contrast, multiethnic analyses, such as this study, not only enhance generalizability, but may 319 also boost power for certain traits, particularly in contexts of limited allelic heterogeneity. The 
320 possibility of false positive loci is also a consideration, given the absence of external replication.

321 However, all newly identified loci passed FDR $<1 \%$. Such statistical limitations are further

322 mitigated in the case of $A T X N 1$ and UBE2E2 by functional validation of these loci in murine 323 adipose tissue.

324 Combining large-scale discovery human genetics with the detailed fat phenotyping and 325 experiments in model organisms identified 7 new loci in association with ectopic fat traits, of 326 which $A T X N 1$ and UBE2E2 demonstrated a functional effect during adipocyte differentiation.

327 Future studies should further explore the exact mechanism by which modulation of ATXN1 and 328 UBE2E2 impact adipocyte differentiation and whether this effect causally impacts systemic 329 metabolic disease.

330

331 
Data availability statement: Summary statistics for all meta-analyses will be made available at the following website https://www.nhlbi.nih.gov/research/intramural/researchers/ckdgen.

Acknowledgements: Please see Supplementary Note for Acknowledgments and Funding Sources.

\section{Author contributions}

Study design: X Guo, AH Kissebah, J Kullberg, LJ Launer, M Olivier, PA Peyser, IB Borecki,

DW Boden, SA Czerwinski, J Ding, V Gudnason, TB Harris, C Langefeld, L Lind, Y Liu, JI

Rotter, B Towne, M Allison

Study management: Yi Zhang, LJ Launer, M Olivier, PA Peyser, JG Terry, IB Borecki, DW

Boden, JJ Carr, SA Czerwinski, V Gudnason, TB Harris, L Lind, BD Mitchell, TH Mosely, Jr, JI

Rotter, AR Shuldiner, H Völzke, JG Wilson, M Allison

Subject recruitment: AH Kissebah, J Kullberg, MK Wojczynski, DW Boden, SA Czerwinski, V

Gudnason, L Lind, BD Mitchell, TH Mosely, Jr, AR Shuldiner, B Towne, H Völzke

Interpretation of results: AY Chu, X Deng, VA Fisher, Yang Zhang, MF Feitosa, C Liu, O Weeks, AC Choh, Q Duan, X Guo, NL Heard-Costa, X Liu, L Lu, JR O'Connell, A Parihar, AV Smith, Yi Zhang, AH Kissebah, M Olivier, PA Peyser, JG Terry, MK Wojczynski, LF Bielak, IB

Borecki, DW Boden, JJ Carr, SA Czerwinski, J Ding, N Friedrich, SL Kardia, C Langefeld, Y Liu, BD Mitchell, JI Rotter, AR Shuldiner, B Towne, H Wallaschofski, M Allison, CM Lindgren, W

352 Goessling, LA Cupples, ML Steinhauser, CS Fox

353 Drafting manuscript: AY Chu, Yang Zhang, MF Feitosa, X Guo, JW Kent Jr., Yi Zhang, AH

354 Kissebah, MK Wojczynski, IB Borecki, CM Lindgren, ML Steinhauser, CS Fox

355 Critical review: AY Chu, X Deng, VA Fisher, MF Feitosa, C Liu, O Weeks, AC Choh, X Guo, NL

356 Heard-Costa, JW Kent Jr., X Liu, L Lu, A Mahajan, JR O'Connell, A Parihar, Yi Zhang, G

357 Homuth, AH Kissebah (deceased), J Kullberg, M Nauck, M Olivier, PA Peyser, JG Terry, LF 
Bielak, J Blangero, IB Borecki, DW Boden, JJ Carr, SA Czerwinski, J Ding, N Friedrich, E Ingelsson, SL Kardia, C Langefeld, L Lind, Y Liu, BD Mitchell, AP Morris, TH Mosely, Jr, JI Rotter, AR Shuldiner, B Towne, H Völzke, H Wallaschofski, M Allison, CM Lindgren, W Goessling, LA Cupples, ML Steinhauser, CS Fox

Statistical methods and analysis: AY Chu, X Deng, VA Fisher, A Drong, Yang Zhang, MF

363 Feitosa, AC Choh, Q Duan, TD Dyer, JD Eicher, X Guo, NL Heard-Costa, T Kacprowski, JW

364 Kent Jr., LA Lange, X Liu, K Lohman, L Lu, A Mahajan, JR O'Connell, A Parihar, JM Peralta, AV

365 Smith, J Yao, LF Bielak, J Ding, C Langefeld, Y Liu, BD Mitchell, AP Morris, CM Lindgren

366 Genotyping: Yi Zhang, G Homuth, M Olivier, DW Boden, SA Czerwinski, E Ingelsson, SL

367 Kardia, Y Liu, AP Morris, JI Rotter, AR Shuldiner, B Towne, CM Lindgren

368 Bioinformatics: AY Chu, X Deng, VA Fisher, MF Feitosa, C Liu, AC Choh, JD Eicher, AD

369 Johnson, T Kacprowski, AV Smith, Yi Zhang

370 Data collection: Yang Zhang, O Weeks, R Laqua, N Friedrich, W Goessling, ML Steinhauser

371 Animal work/functional data: Yang Zhang, ML Steinhauser

373 Disclosures: Caroline S. Fox and Audrey Y. Chu are employed by Merck Research

374 Laboratories as of December 14, 2015 and July 18, 2016, respectively.

376 Disclaimer: The views expressed in this manuscript are those of the authors and do not 377 necessarily represent the views of the National Heart, Lung, and Blood Institute; the National 378 Institutes of Health; or the U.S. Department of Health and Human Services. Please see 379 Supplementary Note for acknowledgements and funding sources. 
383 1. Ding, J. et al. The association of regional fat depots with hypertension in older persons 
20. Baba, S., Jacene, H. A., Engles, J. M., Honda, H. \& Wahl, R. L. CT Hounsfield units of brown adipose tissue increase with activation: preclinical and clinical studies. J. Nucl. Med. 51, 246-250, doi:10.2967/jnumed.109.068775 (2010).

21. Hu, H. H., Chung, S. A., Nayak, K. S., Jackson, H. A. \& Gilsanz, V. Differential computed tomographic attenuation of metabolically active and inactive adipose tissues: preliminary findings. J. Comput. Assist. Tomogr. 35, 65-71, doi:10.1097/RCT.0b013e3181fc2150 (2011).

22. Heid, I. M. et al. Meta-analysis identifies 13 new loci associated with waist-hip ratio and reveals sexual dimorphism in the genetic basis of fat distribution. Nat. Genet. 42, 949960, doi:10.1038/ng.685 (2010).

23. Ward, L. D. \& Kellis, M. HaploReg: a resource for exploring chromatin states, conservation, and regulatory motif alterations within sets of genetically linked variants. Nucleic Acids Res. 40, D930-934, doi:10.1093/nar/gkr917 (2012).

24. Boyle, A. P. et al. Annotation of functional variation in personal genomes using RegulomeDB. Genome Res. 22, 1790-1797, doi:10.1101/gr.137323.112 (2012).

25. Replication, D. I. G. et al. Genome-wide trans-ancestry meta-analysis provides insight into the genetic architecture of type 2 diabetes susceptibility. Nat. Genet. 46, 234-244, doi:10.1038/ng.2897 (2014).

26. Yamauchi, T. et al. A genome-wide association study in the Japanese population identifies susceptibility loci for type 2 diabetes at UBE2E2 and C2CD4A-C2CD4B. Nat. Genet. 42, 864-868, doi:10.1038/ng.660 (2010).

27. Hara, K. et al. Genome-wide association study identifies three novel loci for type 2 diabetes. Hum. Mol. Genet. 23, 239-246, doi:10.1093/hmg/ddt399 (2014). 
457 Figure 1. Functional characterization of Atxn1, Ebf1, Rreb1 and Ube2e2.

$458(a, b, e)$ Data is displayed as box/whisker plots where the center line represents the median, box 459 limits contain the $25^{\text {th }}-75^{\text {th }}$ percentiles, and whiskers span max/min values.

460 (a) Gene expression measured by qPCR in murine subcutaneous (SAT), perigonadal visceral 461 (VAT), and pericardial (PAT) adipose tissues ( $n=6$ mice). Statistical significance was assessed 462 using ANOVA and Sidak's correction for multiple comparisons.

463 (b) Gene expression measured by qPCR in murine adipose tissues after 8 weeks of high fat 464 feeding compared to normal chow fed controls ( $n=5$ mice per group). Statistical significance was 465 assigned using a two-sided T-test.

466 (c) Gene expression measured by qPCR in cultured adipocyte progenitors isolated from the 467 subcutaneous (SAT) or perigonadal visceral (VAT) depots ( $n=4$ replicates). Cells were 468 expanded to confluence and then collected at intervals after induction of adipogenic 469 differentiation. Data displayed as mean, error bar=s.e.m. Statistical significance was assessed 470 using ANOVA and Sidak's correction for multiple comparisons to time 0.

471 (d) Oil-red-o staining of progenitors isolated from subcutaneous adipose and exposed to

472 retroviral delivery of shRNA constructs during ex vivo expansion and induction of adipogenesis.

473 Relative to control vector carrying a scramble sequence, shRNA constructs specific for Atxn1

474 and Ube2e2 impaired adipogenic differentiation. Scale=1mm.

475 (e) Oil-red-o stain was alcohol extracted and quantified at $\mathrm{OD}_{520}(\mathrm{n}=9$ technical replicates).

476 Statistical significance was assessed using ANOVA and Sidak's correction for multiple

477 comparisons to control (Scramble). Data representative of 3 independent experiments. 
478 Table 1. SNPs associated with ectopic fat traits $\left(p<5 \times 10^{-8}\right)^{1}$. Association statistics were obtained using a sample-size weighted fixed479 effects meta-analysis implemented in METAL. ${ }^{18,19}$

\begin{tabular}{|c|c|c|c|c|c|c|c|c|c|c|c|c|}
\hline Locus $^{2}$ & Trait & Strata & Lead SNP & Chr & SNPID & Position & $A 1^{3}$ & $A 2^{4}$ & $\begin{array}{l}\text { Freq } \\
A 1^{5}\end{array}$ & $N$ & $\begin{array}{c}Z \\
\text { score }\end{array}$ & $P$-value ${ }^{6}$ \\
\hline \multicolumn{13}{|c|}{ Fat Volume Traits ${ }^{7,8}$} \\
\hline \multicolumn{13}{|c|}{ NEW } \\
\hline ENSA & PATadjHtWt & ALL & rs6587515 & 1 & rs6587515 & 148875512 & a & g & 0.09 & 11027 & -5.94 & $2.8 \times 10^{-9}$ \\
\hline GRAMD3 & VATadjBMI & WOMEN & rs10060123 & 5 & rs10060123 & 125711809 & a & $\mathrm{c}$ & 0.23 & 9623 & 5.47 & $4.5 \times 10^{-8}$ \\
\hline \multirow{2}{*}{$E B F 1$} & PATadjHtWt & ALL & rs1650505 & 5 & rs1650505 & 157962312 & a & $g$ & 0.24 & 11566 & -6.10 & $1.0 \times 10^{-9}$ \\
\hline & PAT & ALL & & 5 & rs2434264 & 157954781 & $\mathrm{t}$ & $g$ & 0.61 & 11614 & 5.93 & $3.0 \times 10^{-9}$ \\
\hline RREB1 & VATadjBMI & ALL & rs2842895 & 6 & rs2842895 & 7051315 & c & $g$ & 0.50 & 17297 & 5.72 & $1.1 \times 10^{-8}$ \\
\hline GSDMB & SAT & WOMEN & rs2123685 & 17 & rs2123685 & 35307415 & $\mathrm{t}$ & c & 0.94 & 7137 & 5.52 & $3.4 \times 10^{-8}$ \\
\hline \multicolumn{13}{|l|}{ KNOWN } \\
\hline \multirow{4}{*}{ TRIB2 } & PATadjHtWt & ALL & \multirow{4}{*}{ rs10198628 } & \multirow{4}{*}{2} & \multirow{4}{*}{ rs10198628 } & \multirow{4}{*}{12881948} & \multirow{4}{*}{ a } & \multirow{4}{*}{9} & 0.42 & 11572 & -8.88 & $6.7 \times 10^{-19}$ \\
\hline & PATadjHtWt & MEN & & & & & & & 0.43 & 5466 & -6.68 & $2.4 \times 10^{-11}$ \\
\hline & PATadjHtWt & WOMEN & & & & & & & 0.42 & 6106 & -6.02 & $1.8 \times 10^{-9}$ \\
\hline & PAT & ALL & & & & & & & 0.42 & 11605 & -7.87 & $3.7 \times 10^{-15}$ \\
\hline FTO & SAT & ALL & rs7185735 & 16 & rs7185735 & 52380152 & a & g & 0.58 & 17812 & -6.05 & $1.4 \times 10^{-9}$ \\
\hline \multicolumn{13}{|c|}{ Fat Attenuation Traits ${ }^{7,8}$} \\
\hline \multicolumn{13}{|c|}{ NEW } \\
\hline ATXN1 & SATHU & MEN & rs2237199 & 6 & rs2237199 & 16538000 & a & $g$ & 0.11 & 5780 & 5.67 & $1.4 \times 10^{-8}$ \\
\hline \multicolumn{13}{|c|}{ Relative Fat Distribution Traits ${ }^{7,8}$} \\
\hline \multicolumn{13}{|c|}{ NEW } \\
\hline \multirow{2}{*}{ UBE2E2 } & VAT/SAT ratio & ALL & \multirow{2}{*}{ rs7374732 } & \multirow{2}{*}{3} & \multirow{2}{*}{ rs7374732 } & \multirow{2}{*}{23178458} & \multirow{2}{*}{$\mathrm{t}$} & \multirow[b]{2}{*}{ C } & 0.69 & 18205 & -6.29 & $3.1 \times 10^{-10}$ \\
\hline & VAT/SAT ratio adjBMI & ALL & & & & & & & 0.69 & 18190 & -5.64 & $1.7 \times 10^{-8}$ \\
\hline \multicolumn{13}{|l|}{ KNOWN } \\
\hline \multirow{2}{*}{ LYPLAL1 } & VAT/SAT ratio & ALL & \multirow{2}{*}{ rs6689335 } & 1 & rs6689335 & 217695305 & $\mathrm{t}$ & c & 0.59 & 15214 & -5.59 & $2.3 \times 10^{-8}$ \\
\hline & VAT/SAT ratio adjBMI & ALL & & 1 & rs6689335 & 217695305 & $\mathrm{t}$ & c & 0.59 & 15199 & -5.53 & $3.2 \times 10^{-8}$ \\
\hline \multirow{2}{*}{ LY86 } & VAT/SAT ratio & ALL & \multirow{2}{*}{ rs912056 } & \multirow{2}{*}{6} & \multirow{2}{*}{ rs912056 } & 6681196 & & & 0.35 & 17387 & -5.96 & $2.5 \times 10^{-9}$ \\
\hline & VAT/SAT ratio adjBMI & ALL & & & & 0081190 & a & l & 0.35 & 17372 & -5.98 & $2.3 \times 10^{-9}$ \\
\hline
\end{tabular}

$480{ }^{1}$ SNPs are grouped by ectopic fat trait and are listed by new discoveries and then previously identified loci. Any association attaining

481 genome-wide significance $\left(p<5 \times 10^{-8}\right)$ is listed. 
${ }^{2}$ Conventional locus name based on closest gene in the region

$483 \quad{ }^{3} \mathrm{~A} 1$ is the coded allele

$484{ }^{4} \mathrm{~A} 2$ is the non-coded allele

$485 \quad{ }^{5}$ FreqA1 is the allele frequency of Allele1

$486 \quad{ }^{6} \mathrm{P}$-values are double genomic control corrected

$487{ }^{7}$ European and African ancestry cohorts contributed to all ectopic fat traits; Chinese and Hispanic ancestry cohorts contributed only

488 to pericardial volume traits

$489{ }^{8}$ Abbrevations:

SAT - Subcutaneous Adipose Tissue Volume

VAT - Visceral Adipose Tissue Volume

PAT - Pericardial Adipose Tissue Volume

SATHU - Subcutaneous Adipose Tissue Attenuation

VATHU - Visceral Adipose Tissue Attenuation

VAT/SAT ratio - Visceral to Subcutaneous Adipose Tissue Volume Ratio

adjBMI - Model Adjusted for BMI

adjHtWt - Model Adjusted for Height and Weight 
Figure 1.

a

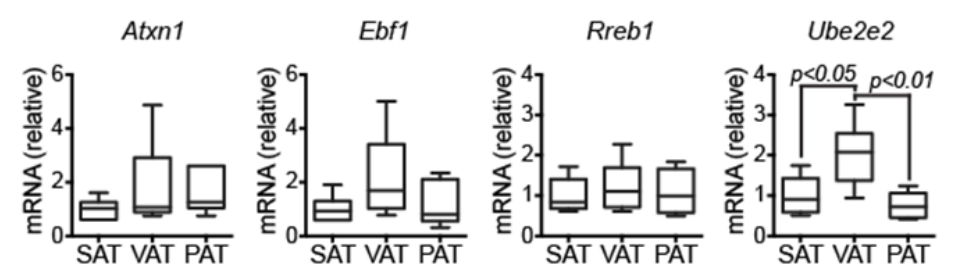

b 吕Chow

Atxn1

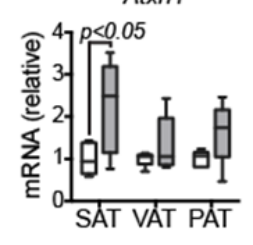

Ebf1
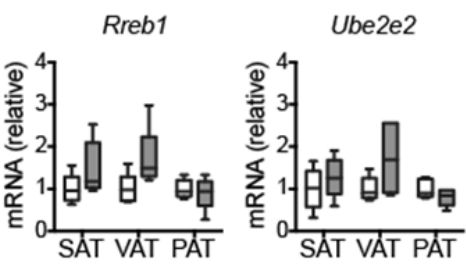

d

(-) control

sh-Scramble

Adipogenic induction

$$
\text { sh-Atxn1 }
$$

sh-Ube2e2

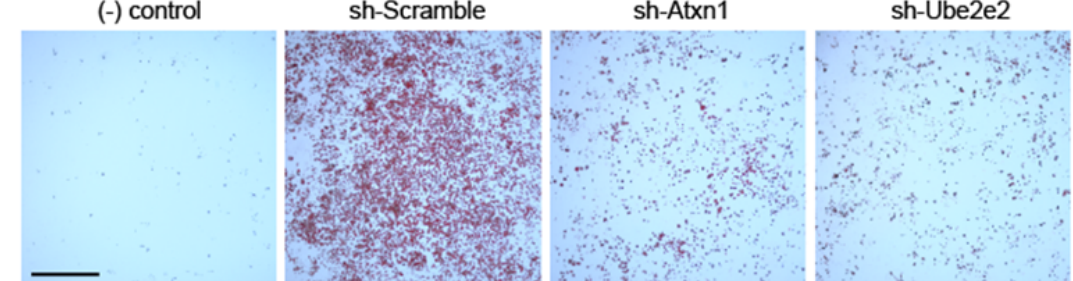

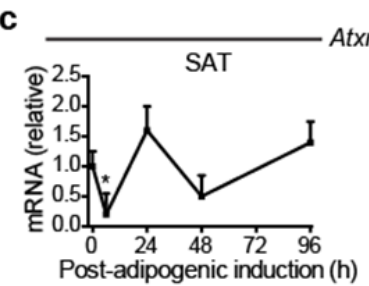

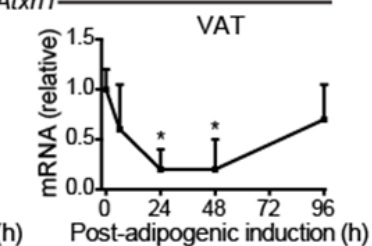

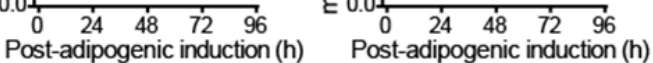
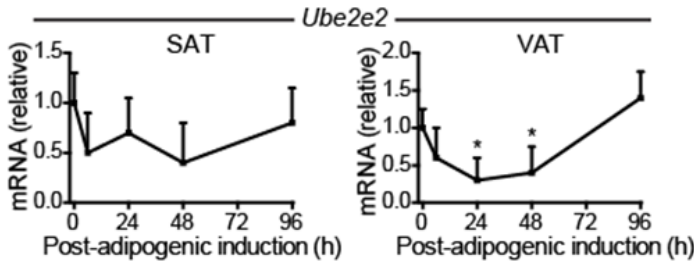

\section{e}
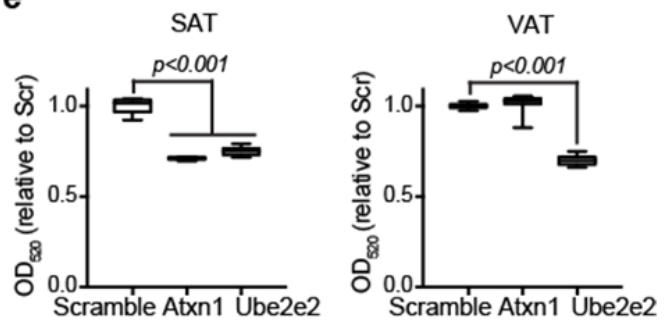


\section{Online Methods}

501 Study Participants

Up to 18,332 participants from 13 cohorts of European and African ancestry were

503 available for analysis of subcutaneous and visceral adipose tissue volumetric traits, up to

504 11,596 from 6 cohorts of European, African, Asian, and Hispanic ancestry were available for

505 analysis of pericardial adipose volumetric traits, up to 12,519 participants from 5 cohorts of

506 European and African ancestry were available for analysis of attenuation traits, and up to

50718,191 participants from 6 cohorts of European and African ancestry were available for analysis

508 of relative fat distribution traits. This epidemiological sample constitutes the largest known

509 collection of participants with radiologically derived ectopic fat measures and genetic data at the

510 inception of this project. Supplementary Table 2 and 3 contain information regarding imaging

511 modality used by each cohort, distribution by sex and ancestry per cohort for each trait analyzed

512 and cohort descriptive information. All participants provided informed consent and each study

513 was approved by their governing ethics committee.

$515 \quad$ Trait assessment

516 The traits measured in this study can be categorized into three groups: 1) fat volume

517 measurements: subcutaneous adipose tissue (SAT), visceral adipose tissue (VAT) and

518 pericardial adipose tissue (PAT); 2) fat attenuation measurements: subcutaneous adipose

519 tissue attenuation (SATHU) and visceral adipose tissue attenuation (VATHU); and 3) relative fat

520 distribution measurements: visceral-to-subcutaneous adipose tissue volume ratio (VAT/SAT

521 ratio). All volume-based measures were assessed by computed tomography (CT) or magnetic

522 resonance imaging (MRI) following study-specific protocols; attenuation-based measures were

523 assessed by CT following study specific protocols. Please see Supplementary Table 2 and

524 Supplementary Note for further detail. 
The following traits were created by each cohort in the overall sample, women and men:

526 volume-based traits - SAT, VAT, VAT adjusted for BMI, PAT, PAT adjusted for height and

527 weight; attenuation-based traits - SATHU and VATHU; relative-distribution traits - VAT/SAT

528 ratio, VAT/SAT ratio adjusted for BMI pericardial traits. The rationale for including the ectopic

529 fat traits, the adjustment models, and the sex-stratified analyses was 4-fold. First, ectopic fat

530 measures are correlated with each other and with general adiposity and we wished to adjust for

531 these factors as potential confounders or intermediates and to examine the genetic associations

532 independent of the adjustment factor. Please see refer to Supplementary Table 7 for pairwise

533 correlations of all traits within FHS, the largest participating cohort. For example, the correlation

534 between VAT and BMI is 0.71 to 0.75 and adjusting for BMI when examining VAT provides the

535 relative amount of VAT controlling for degree of general adiposity. Although the correlations

536 between VAT/SAT ratio and BMI are modest, adjusting for BMI allowed us to examine the

537 propensity to store fat viscerally compared to subcutaneously independent of general adiposity.

538 Second, adjustment of covariates reduces the residual variance of the trait associated with the

539 given covariate and thus increases power to detect genetic associations. Third, in the adiposity

540 genetics literature there is evidence of sexually dimorphic loci in which the variance explained is

541 larger in women versus $\operatorname{men}^{28}$ and association of the loci is markedly stronger in women

542 compared to men, and vice versa. ${ }^{14,22}$ Lastly, we adjusted PAT for height and weight to be

543 consistent with our prior work ${ }^{13}$ (see Supplementary Table 1 for guide to nomenclature for traits

544 and adjustment models).

545 Due to the known differences in body fat distribution by sex, each cohort created sex-

546 and ancestry-specific residuals adjusted for age, age-squared, smoking status, measures of

547 subpopulation stratification and family structure (if necessary). Family-based studies created an

548 additional set of residuals from all participants (both women and men) to account for family

549 structure when analyzing the overall sample. Participants with missing genotype, phenotype or

550 covariate data were excluded from analysis as pre-specified in the analysis plan. 
Study Specific Protocol

Trait measurements and descriptions from each cohort are available in Supplementary

553 Material under "Cohort Specific Information and Protocols".

\section{Genotyping and Imputation}

Each cohort was genotyped as specified in Supplementary Table 4 and performed

557 ancestry-specific imputation up to $\sim 2.6$ million SNPs based on the HapMap Project Phase 2

558 haplotypes (http://hapmap.ncbi.nlm.nih.gov/index.html.en). All newly identified loci were

559 imputed with imputation qualities $>0.8$ in each cohort. Imputation quality by locus and cohort

560 are available in Supplementary Table 8.

\section{Heritability Analysis}

Heritability was estimated from the Framingham Heart Study using variance components

564 analysis in SOLAR. ${ }^{16}$

565

Genetic Correlation Analysis

Pairwise genetic correlations between subcutaneous fat (volume and attenuation),

568 visceral fat (volume and attenuation), ratio of visceral-to-subcutaneous fat and BMI were 569 calculated using SOLAR ${ }^{16}$ in the Framingham Heart Study among 3,312 participants. We used

570 residuals adjusted for age and sex. Two separate hypotheses were tested: 1) RhoG=0 is the

571 test for overlapping genetic correlations, and 2) absolute value (RhoG) $=1$ is the test for non-

572 overlapping genetic correlations.

\section{$574 \quad$ Statistical Analysis}

575 Within each cohort, by ancestry and by sex, genome-wide linear regression analyses 576 were conducted on the 11 trait and model combinations assuming an additive genetic model 
577 using allele dosages. All traits approximated a normal distribution and untransformed traits

578 were used for analysis. To prevent the undue influence of rare variants and/or of poorly

579 imputed SNPs, we included variants with a minor allele count $>10$ and imputation quality $>0.4$

580 (for $\mathrm{MaCH}^{29}$ ) or $>0.3$ (for IMPUTE ${ }^{30}$ ) in each cohort.

$581 \quad$ For multiethnic analysis, we combined all cohort-specific results using a sample size-

582 weighted fixed-effects meta-analysis (Stouffer's method) as implemented in METAL ${ }^{18,19}$ to allow

583 for differences in trait measurement and scaling due to different imaging modalities across

584 cohorts. European and African ancestry cohorts contributed to all ectopic fat traits; Chinese and

585 Hispanic ancestry cohorts contributed only to pericardial volume traits (Supplementary Table 3).

586 All analyses were performed for the overall sample (ALL), among women only (WOMEN) and

587 among men only (MEN). All analyses were corrected for genomic control at the cohort-level.

588 We excluded variants with minor allele frequency (MAF) $<5 \%$ due to the low power to detect

589 associations of such variants. We set a traditional genome-wide significance threshold at

$590 \mathrm{P}<5 \times 10^{-8}$, the Bonferroni correction for the number of independent and common variants across

591 the genome ( 1 million SNPs). All p-values represent two-sided p-values unless otherwise

592 specified. All regional association plots, Manhattan plots, and QQ plots were created using $\mathrm{R}$

593 version 3.1.1 (https://cran.r-project.org/). Linkage disequilibrium plots were created using

594 SNAP ${ }^{31}$ and the gap R package (https://www.jstatsoft.org/article/view/v023i08).

$595 \quad$ To correct for multiple testing, false discovery rate (FDR) was calculated across the 27

596 ectopic fat GWAS scans using the qvalue R package (http://github.com/jdstorey/qvalue).

597 FDR $<1 \%$ was set as the multiple testing corrected significance threshold.

$598 \quad$ For mouse studies, individual cages of mice were randomly assigned in an un-blinded

599 fashion to normal chow or high fat diet. Each in vivo study was conducted one time and no mice

600 were excluded from the analyses. In the absence of a priori data regarding the variance of gene

601 expression in the tissues of interest, we applied sample sizes that have in our experience been

602 of sufficient size to detect a two-fold increase in gene expression. For normally distributed data 
603 from more than two groups (Shapiro-Wilk), an ANOVA test followed by Sidak's correction for

604 multiple testing was conducted (Figures 1a,c,e). For non-normal data a Kruskal-Wallis test was

605 used. For comparisons between two normally distributed groups (Figure 1b: chow versus high

606 fat) a two-sided T-test was used, unless the data was non-normal, in which case a Mann-

607 Whitney test was used. Data were expressed as mean, s.e.m. Significance was assigned for

608 two-sided $p<0.05$. Data were analyzed and graphed using JMP 10.0 (SAS institute) and Prism 6

609 (Graphpad).

610

611 Sensitivity Analyses

612 To ensure the newly identified loci from our multiethnic analysis were robust and not

613 driven by statistical outliers related to ancestry, ancestry-specific meta-analysis results were

614 compared with each other with respect to the minor allele, the minor allele frequency and

615 direction of the Z-score association statistic (Supplementary Table 9). Due to the scaling

616 differences in imaging modalities across each cohort and use of the sample size weighted meta-

617 analysis heterogeneity statistics cannot be calculated.

618 The lead SNP for the GSDMB locus associated with SAT in women was not observed in

619 non-European ancestry cohorts and thus was not included in this analysis. For each of the

620 remaining 6 lead SNPs from the newly identified ectopic fat loci, Z scores were directionally

621 consistent across ancestry-specific meta-analyses (please see Supplementary Figure 2 for

622 forest plots of each locus and Supplementary Figure 3 for linkage disequilibrium [LD] plots

623 across ancestry). For 5 of these loci, the minor allele was identical across ancestries; only the

624 minor allele of rs2842895 (RREB1) differed between the European ancestry and African

625 ancestry cohorts. This observation may explain the slight attenuation in the association of

626 RREB1 and VATadjBMI after combining European and African ancestries in the multiethnic

627 meta-analysis $\left(P_{\text {European-ancestry }}=5.8 \times 10^{-9}\right.$ to $\left.P_{\text {multiethnic }}=1.1 \times 10^{-8}\right)$, although the multiethnic result

628 remains genome-wide significant. 
For each SNP attaining genome-wide significance in association with any ectopic fat

631 trait, we extracted association results in each strata of analysis (ALL, WOMEN, and MEN) for 632 related ectopic fat traits within our study.

633 To investigate the association of the new ectopic fat loci with measures of generalized

634 adiposity (BMI) and central obesity (WHR) - two traits that are strongly correlated with, but

635 distinct from ectopic fat - we evaluated the lead genome-wide significant SNPs in publically

636 available datasets from the most recent GIANT meta-analyses of BMI and WHR. ${ }^{14,15}$

637 To investigate associations of new loci with cardio-metabolic traits that are

638 epidemiologically associated with ectopic fat, cross-trait evaluations for the lead SNPs only were 639 performed in the publically available datasets from the MAGIC (Meta-Analyses of Glucose and

640 Insulin Consortium for fasting glucose and insulin ${ }^{32}$ ), GLGC (Global Lipids Genetics Consortium

641 for high-density lipoprotein cholesterol, triglycerides and total cholesterol ${ }^{33}$ ),

642 CARDIoGRAM+CAD consortium (Coronary ARtery Dlsease Genome wide Replication and

643 Meta-analysis [CARDIoGRAM] plus The Coronary Artery Disease [C4D] Genetics for coronary

644 artery disease and myocardial infarction ${ }^{34,35}$ ), ICBP (International Consortium for Blood

645 Pressure for systolic and diastolic blood pressure ${ }^{36}$ ), and DIAGRAM (DIAbetes Genetics

646 Replication And Meta-analysis ${ }^{25}$.

Analysis of general adiposity and central adiposity loci

To evaluate the relationship between the known $97 \mathrm{BMI}$ and $49 \mathrm{WHR}$ loci ${ }^{14,15}$ with

650 ectopic fat traits, we examined the association for these loci with fat volume and relative fat

651 volume traits among the combined multiethnic sample of women and men. Because the ectopic

652 fat data may be underpowered to determine statistically significant results, we hypothesized that

653 the direction of the BMI and WHR findings would be directionally consistent with the ectopic fat

654 traits, even if the p-values were not significant. Binominal tests were used to test the 
655 significance of direction consistent associations (1-sided p-values). If the binominal test across

656 the BMI or WHR loci was significant, a second 1-sided binominal test was performed evaluating 657 consistency of associations restricting to SNPs with nominally significant associations $(P<0.05)$.

Functional Profiling - Bioinformatics and Annotation

To further characterize novel genome-wide significant loci, the following bioinformatics

661 databases were queried for the lead ectopic fat loci: GWAS Catalog

662 (https://www.ebi.ac.uk/gwas/; access date: 10/15/2015) to investigate other traits associated

663 with newly identified loci, and HaploReg ${ }^{23}$ and RegulomeDB ${ }^{24}$ to identify regulatory elements

664 overlapping the loci for the index SNP and SNPs in LD with the index SNP $\left(r^{2}>0.8\right.$;

665 Supplementary Table 13). To contextualize the newly identified ectopic loci and the surrounding

666 genes, SNIPPER (https://github.com/welchr/Snipper.git) was used to search for biologically

667 relevant mechanisms (Supplementary Table 14).

668

$669 \quad$ Variance Explained

670 The variance explained for each of the loci was approximated using the following

671 formula $R^{2}=\beta^{2} \operatorname{var}(S N P) / v a r\left(e c t o p i c\right.$ fat trait), where $\beta^{2}$ is the estimated effect of the SNP on the

672 ectopic fat trait, and $\operatorname{var}(S N P)=2^{*} M A F_{S N P}{ }^{*}\left(1-M A F_{S N P}\right)$. Because sample-size weighted fixed-

673 effect meta-analysis does not estimate effect sizes, the beta-coefficient for the association

674 between the SNP and ectopic fat trait and the variance of the ectopic fat trait were obtained

675 from cohort level analysis per contributing study. The mean of the variance explained per locus

676 across all contributing cohorts ranges from $0.1 \%$ to $4.4 \%$ (Supplementary Table 15).

677

678

679 
Power for discovery in the ectopic fat genomewide scan was calculated using

682 GWAPower ${ }^{37}$ using the range of sample size in this study (5,842-18,332 participants) and 683 setting $\alpha=5 \times 10^{-8}$. For the smallest sample size analyzed $(\mathrm{N}=5,842)$ we had $\geq 80 \%$ power to 684 detect loci explaining at least $0.64 \%$ of the trait variance. For the largest sample size analyzed $685(\mathrm{~N}=18,332)$, we had $\geq 80 \%$ power to detect loci explaining at least $0.20 \%$ of the trait variance. 686 For example, our novel loci explained from $0.15-4.4 \%$ of the trait variance for ectopic fat as seen 687 in Supplementary Table 15.

To address the power to detect associations for the lookup analyses, we used 689 GWAPower ${ }^{37}$ with the maximum sample sizes from the each of the quantitative trait datasets 690 (52,000-94,000 participants), a modest range of variance explained (0.01-0.05\%; based on the 691 variance explained for each locus [0.1-4.4\%] and the age- adjusted correlations between 692 ectopic fat and the cardiometabolic trait of interest $\left.\left[\mathrm{R}^{2}=0.02-0.46\right]\right)$ and a Bonferroni corrected $\alpha$

$693=7.4 \times 10-4(\sim 0.05 / 66$ pairs of SNP-trait associations). For the smallest dataset (Fasting Insulin, $694 \mathrm{~N} 52,000$ ), we had $80 \%$ power to detect loci explaining at least $0.030 \%$ of the variance in 695 fasting insulin. For the largest dataset (HDL-C and total cholesterol, N 94,000), we had $80 \%$ 696 power to detect loci explaining $0.018 \%$ of the variance in HDL-C or total cholesterol. These 697 calculations indicate that we largely had adequate power for a large portion of the SNP-trait 698 associations. eQTL analysis Using a curated collection of 6 eQTL datasets in adipose-related tissues, index SNPs at 702 newly identified ectopic fat loci were examined in association with transcript expression.

703 Datasets were collected through publications, publically available sources, or private 704 collaboration. The eQTL datasets met criteria for statistical thresholds for SNP-gene transcript 
705 associations as described in the original papers and were limited to index SNPs and SNPs in

706 LD with the index SNP $\left(r^{2}>0.8\right)$ across all ancestries available in the 1000 Genomes Project pilot

$707\left(\mathrm{SNAP}^{31}\right)$. A general overview of the larger collection of more than 50 eQTL studies from which

708 the adipose-related datasets (omental, visceral and subcutaneous adipose, ${ }^{38-42}$ ) were derived

709 from has been published. ${ }^{43}$ Additional eQTL data was integrated from online sources including

710 ScanDB, the Broad Institute GTEx Portal, and the Pritchard Lab (eqtl.uchicago.edu). Results for

711 GTEx Analysis V4 for subcutaneous adipose tissue were downloaded from the GTEx Portal and

712 then additionally filtered as described below (www.gtexportal.org ${ }^{41}$ ). Splicing QTL (sQTL) results

713 generated with sQTLseeker with false discovery rate $\mathrm{P} \leq 0.05$ were retained. For all gene-level

714 eQTLs, if at least 1 SNP passed the tissue-specific empirical threshold in GTEx, the best SNP

715 for that eQTL was always retained. All gene-level eQTL SNPs with $P<1.67 \times 10^{-11}$ were also

716 retained, reflecting a global threshold correction of $\mathrm{P}=0.05 /(30,000$ genes $X 1,000,000$ tests $)$.

717 Cis-eQTL analysis showed SNPs at ENSA (a locus identified in association with PAT)

718 was correlated with multiple transcripts (MRPS21, CTSK and LASS2, $\mathrm{P}<10^{-4}$ ) in subcutaneous

719 and omental adipose tissue (Supplementary Table 16), suggesting these may be the relevant

720 transcripts at this locus and not ENSA, the closest gene to the lead association signal.

721 However, the ENSA locus was not selected for functional validation, as there were too many

722 genes in the region to practically follow up. No other eQTLs were identified.

723

724 Characterization in Model Organisms

725 Selection of Loci for Characterization

726 For functional follow-up and characterization of ectopic fat loci, four gene-trait

727 associations were selected based on visual examination of regional association plots

728 (Supplementary Figures 1a-g) for a localized association within a gene body at each locus

729 (RREB1, ATXN1 and UBE2E2) or localized association near the gene body and the lack of 
730 other genes within $1 \mathrm{Mbp}$ of the lead SNP (EBF1) to increase the probability of experimentally

731 testing the likely causal gene in murine models.

732

733 Mouse studies

734 Experiments were approved by and in compliance with the ethical regulations of the

735 Harvard Medical Area Standing Committee on Animals. Male C57BL/6 mice were purchased

736 from Charles River and housed at $22 \pm 2^{\circ} \mathrm{C}$, with a $12 \mathrm{~h}$ light $(0700-1900 \mathrm{~h}), 12 \mathrm{~h}$ dark (1900-

$7370700 \mathrm{~h}$ ) cycle and ad libitum access to food and water. With the exception of the data shown in

738 Supplementary Figure 6, experiments were conducted in male mice. Diet-induced obesity was

739 modeled with high fat (D12492) and control chow (D12450J) matched for sucrose content

740 (Research Diets, Inc.). Adipose tissue was harvested, homogenized in Trizol (Life

741 Technologies), and RNA extracted according to the manufacturers protocol. cDNA was

742 synthesized using the High-Capacity cDNA Reverse Transcription Kit (Life Technologies).

743 qPCR was performed using iTaq Universal SYBR Green Supermix (Bio-Rad, Hercules, CA) on

744 an iCycler (Bio-Rad) instrument. See Supplementary Table 17 for primer sequences used in

745 these analyses. Gene expression was normalized to 18S. The delta-delta CT method was

746 utilized to calculate fold change in transcript levels.

747

748 Comparison of baseline adipose-specific expression of Atxn1

749 Given that the SNP-ectopic fat association for ATXN1 was confined to men, we

750 assessed gender-specific effects in mice of Atxn1 expression. There was no detectable gender

751 effect on the baseline, adipose-specific expression of Atxn1 (Supplementary Figure 6).

752

753 Adipogenesis assay

$754 \quad$ Adipose tissue from C57BL/6 mice was minced and digested with collagenase D

755 (Roche) in a shaking water bath (37C, 225rpm, 40min). The digest was centrifuged at 400g for 
$75610 \mathrm{~min}$. Pelleted stromal vascular cells were filtered $(40 \mu \mathrm{m})$ and then washed with PBS and

757 subjected to additional negative selection (CD31- / lineage $\left.{ }^{-}\right)$adapted from previously performed

758 methods ${ }^{44}$ using antibody coated microbeads (Miltenyi Biotec). Cells were cultured to

759 confluence in collagen-coated plates and stimulated with dexamethasone, insulin and 3-

760 isobutyl-1-methylxanthine to induce adipogenic differentiation. For genetic loss of function

761 assays, validated shRNA sequences (Broad, Ube2e2: TRCN0000040962; Atxn1:

762 TRCN0000240655) or scramble sequence were subcloned into a retroviral vector (pMKO.1).

763 Gene knock-down efficiency was confirmed by qPCR in 3T3L1 cells, in each instance

764 reproducibly achieving a minimum of $60 \%$ reduction of transcriptional activity. Differentiation into

765 mature lipid-containing adipocytes was determined by oil-red-o (ORO) staining and quantified

766 by measuring alcohol-extracted ORO dye at optical density $520 \mathrm{~nm}\left(\mathrm{OD}_{520}\right)$.

767

768 Cohort Specific Acknowledgements and Funding

769 Please see the Supplementary Note for acknowledgements and funding statements from 770 all participating cohorts.

771 
773 28. Zillikens, M. C. et al. Sex-specific genetic effects influence variation in body composition.

29. Li, Y., Willer, C. J., Ding, J., Scheet, P. \& Abecasis, G. R. MaCH: using sequence and genotype data to estimate haplotypes and unobserved genotypes. Genet. Epidemiol. 34, 816-834, doi:10.1002/gepi.20533 (2010).

30. Howie, B. N., Donnelly, P. \& Marchini, J. A flexible and accurate genotype imputation method for the next generation of genome-wide association studies. PLoS genetics $\mathbf{5}$, e1000529, doi:10.1371/journal.pgen.1000529 (2009).

31. Johnson, A. D. et al. SNAP: a web-based tool for identification and annotation of proxy SNPs using HapMap. Bioinformatics 24, 2938-2939, doi:10.1093/bioinformatics/btn564 (2008).

32. Manning, A. K. et al. A genome-wide approach accounting for body mass index identifies genetic variants influencing fasting glycemic traits and insulin resistance. Nat. Genet. 44, 659-669, doi:10.1038/ng.2274 (2012).

33. Global Lipids Genetics, C. et al. Discovery and refinement of loci associated with lipid levels. Nat. Genet. 45, 1274-1283, doi:10.1038/ng.2797 (2013).

34. Coronary Artery Disease Genetics, C. A genome-wide association study in Europeans and South Asians identifies five new loci for coronary artery disease. Nat. Genet. 43, 339-344, doi:10.1038/ng.782 (2011).

35. Schunkert, H. et al. Large-scale association analysis identifies 13 new susceptibility loci for coronary artery disease. Nat. Genet. 43, 333-338, doi:10.1038/ng.784 (2011).

36. International Consortium for Blood Pressure Genome-Wide Association, S. et al. Genetic variants in novel pathways influence blood pressure and cardiovascular disease risk. Nature 478, 103-109, doi:10.1038/nature10405 (2011).

37. Feng, S., Wang, S., Chen, C. C. \& Lan, L. GWAPower: a statistical power calculation software for genome-wide association studies with quantitative traits. BMC Genet. 12, 12, doi:10.1186/1471-2156-12-12 (2011).

38. Emilsson, V. et al. Genetics of gene expression and its effect on disease. Nature 452 , 423-428, doi:10.1038/nature06758 (2008).

39. Greenawalt, D. M. et al. A survey of the genetics of stomach, liver, and adipose gene expression from a morbidly obese cohort. Genome Res. 21, 1008-1016, doi:10.1101/gr.112821.110 (2011).

40. Grundberg, E. et al. Mapping cis- and trans-regulatory effects across multiple tissues in twins. Nat. Genet. 44, 1084-1089, doi:10.1038/ng.2394 (2012).

41. Consortium, G. T. The Genotype-Tissue Expression (GTEx) project. Nat. Genet. 45, 580-585, doi:10.1038/ng.2653 (2013).

42. Foroughi Asl, H. et al. Expression quantitative trait Loci acting across multiple tissues are enriched in inherited risk for coronary artery disease. Circ. Cardiovasc. Genet. 8, 305-315, doi:10.1161/CIRCGENETICS.114.000640 (2015).

43. Zhang, X. et al. Synthesis of 53 tissue and cell line expression QTL datasets reveals master eQTLs. BMC Genomics 15, 532, doi:10.1186/1471-2164-15-532 (2014).

44. Kim, S. M. et al. Loss of white adipose hyperplastic potential is associated with enhanced susceptibility to insulin resistance. Cell metabolism 20, 1049-1058, doi:10.1016/j.cmet.2014.10.010 (2014). 